\title{
THE CELESTIAL SYSTEM AND FRAME OF IERS
}

\author{
E.F. Arias (Facultad de Cs. Astronomicas y Geofisicas de La Plata, CONICET, \\ Central Bureau of IERS) \\ M. Feissel (Observatoire de Paris, Central Bureau of IERS)
}

ABSTRACT. The celestial system maintained by the International Earth Rotation Service is described in terms of physical properties of the fiducial objects, internal consistency of the frame, and agreement with the FK5 and dynamical systems.

\section{Introduction}

The celestial reference frame of IERS is based on compact extragalactic objects observed by VLBI. It is maintained on the basis of several independent Earth rotation programs analysed by various analysis centres. We present hereafter its latest realization, which is a combination of individual frames obtained by four groups: GSFC (Ma et al., 1990), JPL (Steppe et al., 1990), NGS (Carter and Robertson, 1990), and USNO (Eubanks et al., 1990). The combination is based on a three rotation angle model applied to a selection of radio sources common to the individual frames. The initial definition of the system and the maintenance process is described by Arias and Feissel (1990); the connection to the conventional terrestrial system of IERS at the level of 0.001 " is studied by Feissel (1990). In this paper, we discuss some aspects of interest to the WGRS: the physical properties of the objects, the precision and internal consistency of the frame, and the definition and maintenance of the system axes.

\section{Physical properties of the fiducial objects}

The distribution on the sky of the latest realization (IERS, 1990) of the IERS celestial reference system is shown on figure 1 ; the declination interval covered is from $-80^{\circ}$ to $+85^{\circ}$; filled circles represent the 51 primary sources.

Figure 1. Distribution of the 228 radio sources of RSC(IERS)90 C 01.

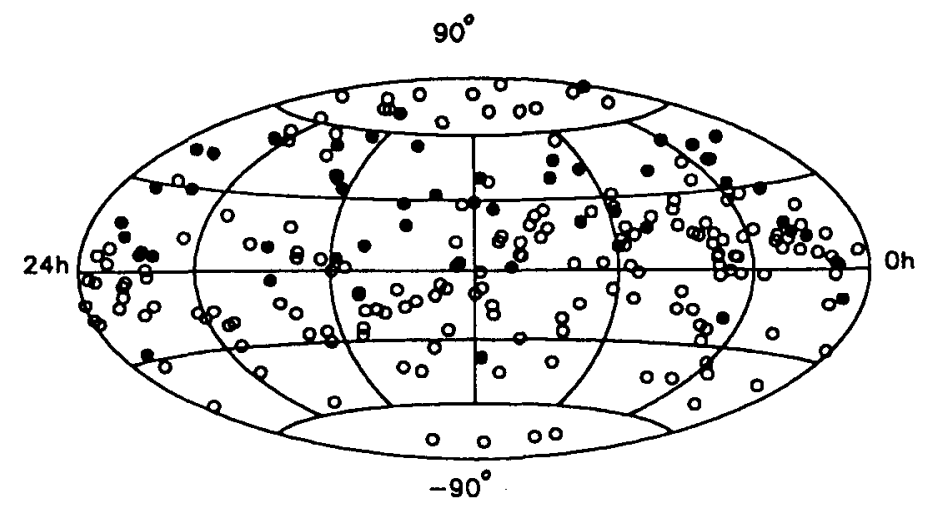


The physical properties of the sources have been investigated on the basis of the VLBI survey published by Preston et al. (1985). This survey covers the full sky; it contains 1398 objects with the following information: redshift (850), total flux (740), spectral index (1200), optical identification (1200), optical magnitude (1000). In addition, the list of IERS sources has been compared to the list of quasars selected for the HIPPARCOS/HST link. Statistics on the type of objects are given in Table 1. The redshifts span the interval 0.1-2.5 quite evenly. The total flux is in general over 1 Jansky, while the survey has a larger proportion of weaker sources. The spectral indices are between -0.8 and +1.4 ; this represents a biasing of the sampling with respect to the complete survey, which has a majority of spectral indices in the interval -1.0 to 0 . The distribution in optical magnitudes is similar to the one of the survey, with a peak around the 18th magnitude. A part of the IERS sources has been mapped at $S$ and $X$ bands (e.g. Charlot 1990). The primary sources mapped show no significant structure at the angular scale of $0.001 "$.

Table 1. Numbers of sources according to their characteristics

\begin{tabular}{crrc}
\hline Characteristics & \multicolumn{2}{c}{ IERS sources } & $\begin{array}{c}\text { Detected in } \\
\text { Survey (917) }\end{array}$ \\
\hline Optical identification & Primary (51) & Other (177) & \\
Quasar & 40 & 115 & 544 \\
BL Lacertae & 5 & 15 & 56 \\
Galaxy & 2 & 8 & 102 \\
Other/unident & 4 & 39 & 215 \\
HIPPARCOS/quasars (95) & 14 & 47 & \\
\hline
\end{tabular}

\section{Internal consistency of the frame}

It is well known that the use of the conventional IAU 1976 Precession and IAU 1980 Theory of Nutation in the analysis of VLBI observations would give rise to systematic errors in the source position, and to the misorientation of the axes of the frames, both at the level of a few milliarcseconds. Therefore the common practice in catalogue work is to estimate additional parameters which describe the motion of the celestial pole relative to its conventional position, either by estimating celestial pole offsets for each session, or by estimating a precession correction and the amplitudes of some of the nutation terms, as allowed by the length and time density of the series of observations analysed. Sovers (1990b) studies in detail the effects of these procedures on the resulting source positions. In the combination performed by the Central Bureau of IERS, only individual frames obtained by one of these procedures are used. The slight offsets between the poles of the catalogues, that are due to inconsistent fixing of the celestial pole offsets at some reference day, are accounted for by the adjustment of rotation angles; they have no influence on the consistency of the individual frames with the combined one.

The noticeably different network geometries of the various observing programs are expected to cause regional deformations in the derived celestial frames. From detailed comparisions of large JPL and GSFC catalogues, Sovers (1990a) concludes that the only systematic difference between the two catalogues is an increase of right ascension differences for lower declinations, amounting to about 0.002 " over the entire range of declinations. This estimation sets an upper limit to the regional errors to be expected in the combined frame.

The realization of the celestial reference system published in the Annual Report of IERS for 1989 contains 228 sources with different status: primary, secondary and 
complementary. The 51 primary sources were chosen on the basis of consistency of their estimated coordinates in the four individual frames, after removing the relative rotations: only sources which showed position differences under $0.0015^{\prime \prime}$ in all comparisons two by two were retained as primary. Their position uncertainties in the IERS frame, derived from this consistency, are smaller than $0.0007 "$. The other sources common to at least two frames but with larger position discrepancies, are considered secondary; there are 40 of them in the realization described here. Finally, 137 complementary sources in the IERS frame were available from only one individual catalogue. Altogether 113 sources have a position uncertainty smaller than 0.001 ", 104 between $0.001 "$ and $0.003 "$, and 11 over $0.003 "$.

\section{Definition and maintenance of the system}

The IERS celestial reference system is barycentric through the appropriate modelling of observations by the analysis centres which contribute individual catalogues. The condition that the sources have no proper motion is also applied by the analysis centres; however, checks are regularly performed to insure the validity of this constraint (Ma, 1990) to avoid spurious (more likely than real) motions of some sources.

The Ox axis was implicetely defined in the initial realization (Arias et al., 1988) by the adoption of the right ascensions of 23 radio sources in catalogues obtained by the GSFC, the JPL, and the NGS. As these catalogues had been compiled by fixing the right ascension of $3 \mathrm{C} 273 \mathrm{~B}$ to the usual conventional FK5 value $(12 \mathrm{~h} 29 \mathrm{~m} 6.6997 \mathrm{~s}$ at J2000.0), the IERS Ox axis is in agreement with the FK5 origin of right ascensions (see Feissel, 1990). In addition, according to Dickey (1989), it is in agreement with the equinox of the JPL planetary frame DE200/LE200 within 0.02".

The $\mathrm{Oz}$ axis points in the direction of the mean pole at $\mathrm{J} 2000.0$ as defined by the IAU conventional models for precession and nutation. As a result of the inaccuracy of the conventional models (Herring, 1990), the Oz axis of the IERS celestial system is shifted from the expected position of the mean pole at J2000.0 by about 0.01 " in longitude $\cdot \sin \varepsilon$ and $0.001^{\prime \prime}$ in obliquity.

The new astrometric techniques and the availability of large computers used to analyze data raise the question of how one should understand the conventional character of the celestial frame. In practice, it can be understood in two ways:

-the source coordinates themselves are considered conventional, i.e., their numerical values are fixed for some time (years); this is the FKn philosophy, -the axes of the system are considered conventionally as fixed to their initial directions, but improved or additional source coordinates available at the time of analysis are provided to the users; this is required for space navigation, Earth orientation programs, geodetic applications, linkage of celestial frames, etc. The system maintenance method applied by the Central Bureau of IERS is along these lines. It should be mentionned that such a procedure is made possible by the high model standardization in the operation of IERS, and by the fact that the implementation of an extragalactic celestial frame implies much less geophysical and astronomical modelling than does the FK series of catalogues.

New realizations of the IERS celestial reference system are produced whenever justified by the progress in the observations or in the modelling. The successive realizations produced up to now have maintained the initial definition of the axes within 0.0001 "; the coordinates of the 51 primary sources have changed by less than $0.0007^{\prime \prime}$ (rms). 


\section{Conclusion: contribution of IERS to astronomy}

The celestial system of IERS was defined and is maintained for the study of the Earth's orientation at a level better than 0.001 ". It contains presently over 100 objects with position uncertainties at this same level.

The contribution of Earth rotation observations to the extragalactic celestial frame includes the monitoring of the sources. In the near future, as a consequence of the extension of the networks in the southern hemisphere for a better coverage of the planet, the sky coverage in the southern hemisphere will be improved, and the systematic errors due to uneven latitude distribution of stations will be diminished. The networks are operated in parallel by groups which tend to extend their lists of observed objects both with common and non common sources, in order to perpetuate their celestial references on a sound basis; this results in a progressive improvement in precision and distribution of the IERS celestial frame.

The physical properties of the fiducial objects will be further investigated in view of the merging into a celestial frame with a larger scope. In its present state, the IERS celestial reference frame has a majority of quasars in the primary sources, an even sampling of redshifts, more than half of the sources having optical counterparts up to the 18 th magnitude, and two-thirds of the HIPPARCOS/HST quasars belonging to it. It can be considered as a valuable contributor to general astronomical references.

\section{References}

Arias, E.F., Feissel, M., Lestrade, J.-F., 1988a. Astron. Astrophys., 199, 357.

Arias, E.F., Feissel, M., Lestrade, J.-F., 1988b. BIH Annual Report for 1987, D-113.

Arias, E.F., Feissel, M.,1990. Proc. IAU Symp. 141, 119, Kluwer.

Carter, W.E., Robertson, D., 1990. IERS Technical Note 5, 25.

Charlot, P., 1990. Astron. Astrophys., 299, 51.

Dickey, J.O., 1989. in Reference Frames in Astronomy and Geophysics, p. 310, Kluwer. Eubanks, T.M., Carter, M.S., Josties, F.J., Matsakis, D.N., McCarthy, D.D., 1990. IERS Technical Note 5, 33.

Feissel, M., 1990, This volume.

Herring, T.A., 1990. This volume.

Ma, C., Ryan, J.W., Caprette, D.S., 1990. IERS Technical Note 5, 1.

Ma, C., 1990. This volume.

Preston, R.A., Morabito, D.D., Williams, J.G., Faulkner, J., Jauncey, D.L., Nicolson, G.D., 1985. Astron. J., 90, 1599.

Sovers, O.J., 1990a. Proc. IAU Symp. 141, 261, Kluwer.

Sovers, O.J., 1990b. This volume.

Steppe, J.A., Oliveau, S.H., Sovers, O.J., 1990. IERS Technical Note 5, 13. 